\title{
Vibration Condition Monitoring: Latest Trend and Review
}

\author{
K.H. Hui ${ }^{1, a}$, L.M. Hee ${ }^{2, b}$, M. Salman Leong ${ }^{3, c}$ and Ahmed M. Abdelrhman ${ }^{4, d}$ \\ ${ }^{1,2}$ RAZAK School of Engineering \& Advanced Technology, Universiti Teknologi Malaysia, Malaysia \\ ${ }^{3,4}$ Faculty of Mechanical Engineering, Universiti Teknologi Malaysia, Malaysia \\ ahuikarhoou@gmail.com, ${ }^{\mathrm{b}} \mathrm{mhlim} @ i c . u t m . m y,{ }^{\mathrm{c}}$ salman@ic.utm.my, ${ }^{\mathrm{d}}$ ahmedrabak@yahoo.com
}

\section{Keywords: Condition Monitoring; Vibration; Condition Monitoring Research.}

\begin{abstract}
Vibration analysis has proven to be the most effective method for machine condition monitoring to date. Various effective signal analysis methods to analyze and extract fault signature that embedded in the raw vibration signals have been introduced in the past few decades such as fast Fourier transform (FFT), short time Fourier transform (STFT), wavelets analysis, empirical mode decomposition (EMD), Hilbert-Huang transform (HHT), etc. however, these is still a need for human to interpret vibration signature of faults and it is regarded as one of the major challenge in vibration condition monitoring. Thus, most recent researches in vibration condition monitoring revolved around using Artificial Intelligence (AI) techniques to automate machinery faults detection and diagnosis. The most recent literatures in this area show that researches are mainly focus on using machine learning techniques for data fusion, features fusion, and also decisions fusion in order to achieve a higher accuracy of decision making in vibration condition monitoring. This paper provides a review on the most recent development in vibration signal analysis methods as well as the AI techniques used for automated decision making in vibration condition monitoring in the past two years.
\end{abstract}

\section{Introduction}

Research in machinery condition monitoring has begun since several decades ago. This is a result of more awareness of the importance of keeping the critical machines such as turbines, pumps, compressors, etc. to run continuously and safely in order to meet its production demands. These critical machines are extensively used in the industries such as in power generation, oil and gas industry, aviation that aim for zero unscheduled downtime. The success of condition monitoring has thus transformed maintenance strategy in certain industries from corrective and preventive to condition-based maintenance [1]. Various condition monitoring and faults diagnosis techniques have been developed such as acoustic emission, pressure, strain gauge measurements, etc. [2] Faults diagnosis by monitoring machine vibration is proven to be the most effective method for machinery condition monitoring. Both the conventional vibration analysis methods such as FFT, STFT and the more recent signal processing methods such as wavelets analysis, EMD, HHT, etc. heavily dependent on the human experience and interpretation of the results. As a result, the current researches in vibration condition monitoring are thus mainly on using AI techniques in order to substitute or reduce the human involvement in results interpretation and decision making in condition monitoring of machines. This review provides a brief discussion of the latest development in these subjects.

\section{Latest Development in Vibration Signal Analysis Methods}

Vibration signal of machines are directly related to the health condition and the working condition of the machines [3]. Latest development in vibration signal analysis has seem the trend of shifting from the frequency based analysis to the time-frequency based analysis, and from the nonadaptive signal analysis to self-adaptive signal analysis. The latest researches in this area are highlighted as follow.

Cong et al. [4] proposed a new signal processing method, namely S-SVDR, which is the shorttime matrix series (STMS) based singular value decomposition (SVD) method using singular value 
ratio (SVR). A new way to create a matrix for SVD process was proposed by the researchers and named as STMS. STMS is capable of extracting fault information from raw vibration signals. SVR spectrum is introduced subsequently by combining both STMS and SVD, and it was reported to have good local identifications for incipient detection for rolling bearings.

Zheng et al. [5] have proposed a new non-stationary signal analysis method namely the local characteristic-scale decomposition (LCD). This method was used to decompose raw vibration signals into a number of intrinsic scale components (ISC) in different scales. LCD is comparable to empirical mode decomposition (EMD) as shown in Fig. 1. Thus, it is more suitable for nonstationary signal analysis purposes. It stated that the LCD is leading in running time, reducing fitting errors, restraining end effect and mode mixing as compared to EMD approach.
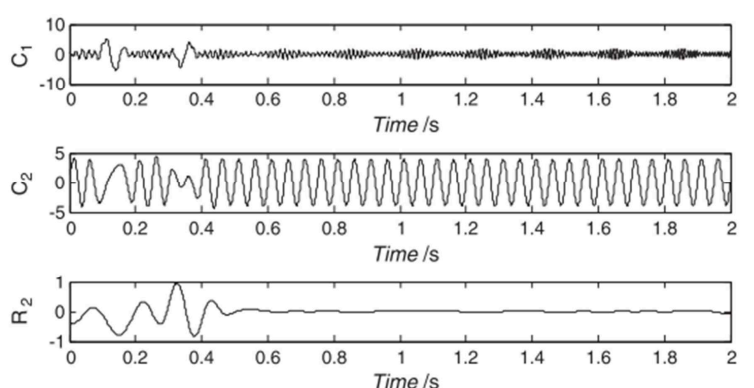

(a)
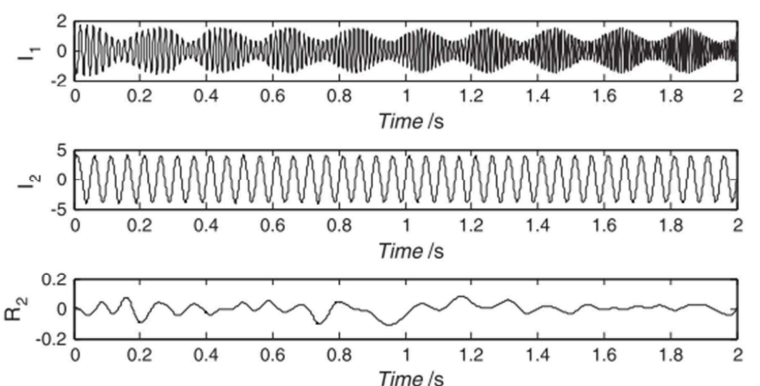

(b)

Fig. 1: The Decomposition Result Generated by (a) EMD (b) LCD [5]

Li et al. [6] proposed a multiscale slope feature extraction method by utilising wavelet-based multiresolution analysis. The proposed method was implemented in three steps: Firstly, the measured vibration signals were processed using discrete wavelet transform (DWT) to obtain a series of detailed signals at different scales. Secondly, the variances of multiscale detailed signals were calculated accordingly. Lastly, the wavelet-based multiscale slope features were estimated from the slope of logarithmic variances. To verify the new proposed method, two experiments; the researchers conducted the bearing defect identification and gear wear diagnosis. The results showed that the proposed method was high in accuracy and stability for machinery fault diagnosis application.

Liu and Han [7] proposed a novel fault feature extraction method based on the local mean decomposition (LMD) and the multi-scale entropy. The researchers conducted a study on faulty rolling bearing. The proposed method decomposed the non-stationary bearing faults signal into a number of product functions. Subsequently, the multi-scale entropy of each product function was calculated, namely feature vectors. It was reported that the newly proposed method is useful for rolling bearing faults feature extraction.

Summary of these newly proposed vibration signal analysis methods is showed in Table 1.

Table 1: Summary of the Recently Published Vibration Signal Analysis Methods

\begin{tabular}{|l|l|l|}
\hline \multicolumn{1}{|c|}{ Author } & \multicolumn{1}{|c|}{ Proposed Vibration Analysis Method } & \multicolumn{1}{c|}{ Application } \\
\hline Cong et al. & $\begin{array}{l}\text { S-SVDR - Short-Time Matrix Series (STMS) based } \\
\text { Singular Value Decomposition (SVD) method using } \\
\text { Singular Value Ratio (SVR) }\end{array}$ & $\begin{array}{l}\text { Rolling bearing } \\
\text { fault diagnosis }\end{array}$ \\
\hline Zheng et al. & Local Characteristic-Scale Decomposition (LCD) & $\begin{array}{l}\text { Rolling bearing } \\
\text { fault diagnosis }\end{array}$ \\
\hline Li et al. & Multiscale slope feature extraction method & $\begin{array}{l}\text { Rotating machinery } \\
\text { fault diagnosis }\end{array}$ \\
\hline Liu and Han & Local Mean Decomposition (LMD) & $\begin{array}{l}\text { Rolling bearing } \\
\text { fault diagnosis }\end{array}$ \\
\hline
\end{tabular}


Review on these papers shows that in general, the latest advancement in vibration signal analysis techniques revolved around methods for signal decomposition. The results were subsequently used for fault features extraction from a complex vibration signal self-adaptively. Physical meaning of the instantaneous frequency (IF) is the major challenge of adaptive time-frequency signal analysis method [5]. The following section discusses AI techniques used in vibration condition monitoring.

\section{Latest Development in Artificial Intelligence Techniques for Vibration Signal Analysis}

AI techniques such as artificial neural networks (ANN), support vector machines (SVM) and Bayesian networks (BN) have been widely adopted in vibration condition monitoring and many other fields. In vibration condition monitoring, AI techniques are widely used for faults classification, data fusion, features reduction, decision making, etc. The latest advancement in AI techniques employed in machinery condition monitoring is discussed as follow.

Chen et al. [8] proposed a novel intelligent fault diagnosis model namely the multi-kernel support vector machine (MSVM) optimised by chaotic particle swarm optimization (CPSO). This method was tested for the diagnosis of roller bearing fault. The multi-kernel function used by the researchers in this study is constructed using Gaussian function and polynomial functions. The benefits of MSVM as reported are it only required small sampling; it has a nonlinearity and high dimension. CPSO is used to determine the optimal parameters for MSVM with high accuracy and great generalization ability. It was reported that the proposed model is superior as compared to single kernel or randomly selected parameters MSVM classifier for faults classification purposes.

Tran et al. [9] applied a new type of machine learning architecture namely the deep belief networks (DBN) to diagnose faults in reciprocating compressor valves. By stacking a number of the restricted Boltzmann machines (RBM) layer by layer, DBN is formed as shown in Fig. 2. One layer of binary stochastic hidden units and one layer of binary stochastic visible units formed the RBM. DBN was reported to be effective for pattern recognition. It was reported that the DBN classifier was capable of improving valve faults identification.

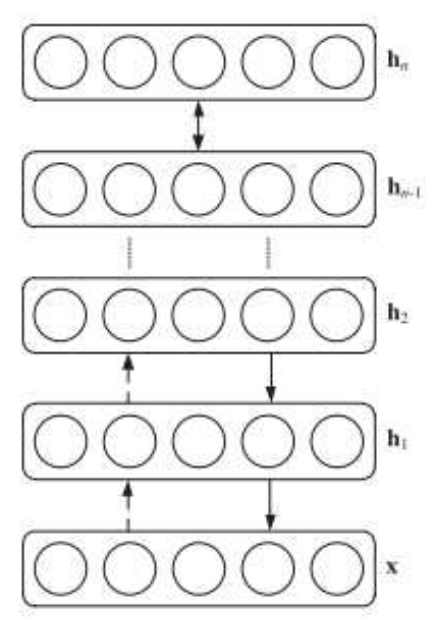

Fig. 2: Architecture of DBN [9]

Zhao et al. [10] proposed the used of trace ratio criterion linear discriminant analysis (TR-LDA) to diagnose rolling element bearing faults. Two new scatter matrices to address rolling element bearing faults dataset with non-Gaussian distribution were developed in the study. The new scatter matrixes were used to characterize the intra-class compactness and the inter-class separability of dataset by local labelled information. The advantages of this method were reported to be able to handle the nonlinear bearing data and also to improve pattern classification performance of faults.

Seera et al. [11] introduced hybrid intelligent models for induction motors condition monitoring. The proposed model combined the fuzzy min-max (FMM) neural network and the random forest (RF) model. The proposed models carried out fault diagnosis in four steps. Firstly, provide input 
data samples. Then, initiate the FMM learning procedure. Subsequently, the hyperboxes generated in the previous step were used as the input samples for classification and regression tree (CART). Lastly, the majority voting scheme used to combine the predictions from an ensemble of trees. The experiment was conducted in both noise-free and noisy environments. Experimental results showed good faults classification performances in both environments.

Summary of the latest development of AI techniques in vibration condition monitoring was shown in Table 2.

Table 2: Summary of the Latest Development of AI Techniques in Vibration Condition Monitoring

\begin{tabular}{|l|l|l|}
\hline \multicolumn{1}{|c|}{ Author } & Latest Researches of Artificial Intelligence Techniques & \multicolumn{1}{c|}{ Application } \\
\hline Chen et al. & $\begin{array}{l}\text { Multi-Kernel Support Vector Machine (MSVM) } \\
\text { optimised by Chaotic Particle Swarm Optimization } \\
\text { (CPSO) }\end{array}$ & $\begin{array}{l}\text { Rolling bearing } \\
\text { fault diagnosis }\end{array}$ \\
\hline Tran et al. & Deep Belief Networks (DBN) & $\begin{array}{l}\text { Reciprocating } \\
\text { compressor valves } \\
\text { fault diagnosis }\end{array}$ \\
\hline Zhao et al. & $\begin{array}{l}\text { Trace Ratio Criterion Linear Discriminant Analysis (TR- } \\
\text { LDA) }\end{array}$ & $\begin{array}{l}\text { Rolling bearing } \\
\text { fault diagnosis }\end{array}$ \\
\hline Seera et al. & $\begin{array}{l}\text { Hybrid intelligent models consist of Fuzzy Min-Max } \\
\text { (FMM) neural network and Random Forest (RF) model }\end{array}$ & $\begin{array}{l}\text { Induction motor } \\
\text { fault diagnosis }\end{array}$ \\
\hline
\end{tabular}

\section{Conclusion}

The utilization of various AI techniques for faults detection and diagnosis was shown to be a major and latest trend in vibration condition monitoring. It is used mainly to reduce human error and to increase the performance of automated faults detection and diagnosis. Researches in this area are anticipated to grow in tandem with the need for more intelligent and more autonomous condition monitoring in the future.

\section{Acknowledgements}

This work is supported by the UTM Flagship Grant (Q.K130000.2409.01G44) and Research University Grant of UTM (Q.K130000.2540.06H51 and Q.J130000.2524.05H01) financed by the Ministry of Education, Malaysia. The main author is joint-funded by the Ministry of Education and Universiti Tun Hussein Onn Malaysia for his Ph.D. study.

\section{References}

[1] K. H. Hui, M. H. Lim, M. S. Leong, and A. M. Abdelrhman, "Time-frequency Signal Analysis in Machinery Fault Diagnosis: Review," in Advanced Materials Research Vol. 845, 2014, vol. 845, pp. 41-45.

[2] A. M. Abdelrhman, M. S. Leong, L. M. Hee, and W. K. Ngui, "Application of Wavelet Analysis in Blade Faults Diagnosis for Multi-Stages Rotor System," Appl. Mech. Mater., vol. 393, pp. 959-964, Sep. 2013.

[3] A. M. Abdelrhman, M. S. Leong, M. H. Lim, and K. H. Hui, "Vibration Analysis of Multi Stages Rotor for Blade Faults Diagnosis," in Advanced Materials Research Vol. 845, 2014, vol. 845, pp. 133-137. 
[4] F. Cong, J. Chen, G. Dong, and F. Zhao, "Short-time matrix series based singular value decomposition for rolling bearing fault diagnosis," Mech. Syst. Signal Process., vol. 34, no. 1-2, pp. 218-230, Jan. 2013.

[5] J. Zheng, J. Cheng, and Y. Yang, "A rolling bearing fault diagnosis approach based on LCD and fuzzy entropy,” Mech. Mach. Theory, vol. 70, pp. 441-453, Dec. 2013.

[6] P. Li, F. Kong, Q. He, and Y. Liu, "Multiscale slope feature extraction for rotating machinery fault diagnosis using wavelet analysis," Measurement, vol. 46, no. 1, pp. 497-505, Jan. 2013.

[7] H. Liu and M. Han, "A fault diagnosis method based on local mean decomposition and multi-scale entropy for roller bearings," Mech. Mach. Theory, vol. 75, pp. 67-78, May 2014.

[8] F. Chen, B. Tang, T. Song, and L. Li, "Multi-fault diagnosis study on roller bearing based on multi-kernel support vector machine with chaotic particle swarm optimization," Measurement, vol. 47, pp. 576-590, Jan. 2014.

[9] V. T. Tran, F. AlThobiani, and A. Ball, "An approach to fault diagnosis of reciprocating compressor valves using Teager-Kaiser energy operator and deep belief networks," Expert Syst. Appl., vol. 41, no. 9, pp. 4113-4122, Jul. 2014.

[10] M. Zhao, X. Jin, Z. Zhang, and B. Li, "Fault diagnosis of rolling element bearings via discriminative subspace learning: Visualization and classification," Expert Syst. Appl., vol. 41, no. 7, pp. 3391-3401, Jun. 2014.

[11] M. Seera, C. P. Lim, S. Nahavandi, and C. K. Loo, "Condition monitoring of induction motors: A review and an application of an ensemble of hybrid intelligent models," Expert Syst. Appl., vol. 41, no. 10, pp. 4891-4903, Aug. 2014. 